Gazi University
Journal of Science
http://dergipark.gov.tr/gujs

\title{
Time Optimal Trajectory Generation with Obstacle Avoidance by Using Optimal Control Theory for a Wheeled Mobile Robot
}

\author{
Masoud MOSAYEBI* (D), Pouya MALLAHI KOLAHI \\ Malek Ashtar University Of Technology, Department of Mechanical Engineering, Iran
}

Highlights

- Two independent actuator are used for path following.

- Using optimal control for path design along obstacle avoidance.

- The minimizing both time and kinetics energy throughout path generation along with obstacle avoidance.

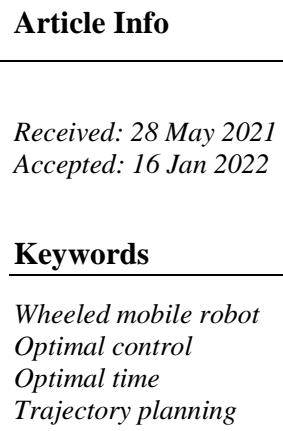

\section{INTRODUCTION}

In the past two decades, wheeled mobile robots (WMRs) have been extensively used in various fields, including factories, military, transportation, and space. During the past decades, the control problem of autonomous WMRs has been investigated. However, recent years have seen an increasing trend toward the design and development of autonomous WMRs using various soft computing techniques. In this regard, the global navigation of the robot, in which the prior awareness of the surrounding spaces should be available, seems to be an essential issue to search for an optimal pathway from the starting point toward the destined point in the presence of fixed obstacles [1]. An important control problem in the WMRs is the presence of nonholonomic constraints as follows [2]:

- In rolling without slipping constraints.

- In systems in which the angular momentum is conserved.

Cui et al. applied adaptive tracking and obstacle avoidance with unknown sliding to control a mobile robot. They are a sliding mode observer, and obstacle avoidance control law is presented to determine the sliding parameters online for nonholonomic systems to compensate for the unknown sliding effects [3]. Furthermore, Nazemzadeh et al. presented an optimum control strategy to plan the optimal trajectory for mobile robots. Through the indirect solution of the optimal control technique, they introduced the trajectory planning of mobile robots [4]. Yen and Cheng introduced an algorithm (fuzzy control with ant colony) that discovers the shortest pathway, distinguishes the entire obstacles found ahead of the robot via ultrasonic transducers, and modifies the mobile robot's turning angles to avoid obstacles [5]. Moreover, they presented an enhanced ant colony algorithm used for WMRs path planning [6]. nazemzadeh et al developed dynamic

* Corresponding author, e-mail: m.mosayebi@mut-es.ac.ir 
equation a mobile robot on slope surface and used nonlinear controllers to track specific paths [7]. Korayem et al. Developed a dynamic model by consider uncertainties and longitudinal and lateral slippage. Considering nonlinear parameters as well as non-holonomic constraints will complicate the process of controlling the moving robot. In order to control a robot, sliding mode control has been used using a Riccati equation to reduce the consumption torque and increase the accuracy of the optimal system [8]. Also in [9] a open-loop controller for the dynamics of industrial robots in order to design the optimal path in order to select the optimal engine in construction presented. Korayem et al. investigated optimal path planning and dynamic modeling of a nonholonomic mobile robot used in complex environments [10]. They did not consider the time parameter in their optimal control problem. Wu et al. introduced an optimum control technique on the basis of the Lyapunov function that is capable of solving a limited input affine nonlinear system for WMRs [11]. In this paper, the global navigation method and the artificial potential field approach are used to fix obstacle avoidance. The time and the kinetic energy quantities will be minimized to generate an optimized trajectory in the presence of a multi-fixed obstacle. Generally, the optimal control problems act on the basis of open-loop control theory. A kinematic equation served as the limitation of the optimal control problem. In section 2, the kinematic equations are derived, and constraints are discussed. In section 3 , the potential function is defined, and then the optimal control problem is determined along with the kinematic constraints. Section 3 presents the problem of optimal control, and a new definition for the Hamiltonian function and the control law is obtained. In Section 4, the simulation and the results are presented. In the following, Figure 1 shows the simulation diagram.

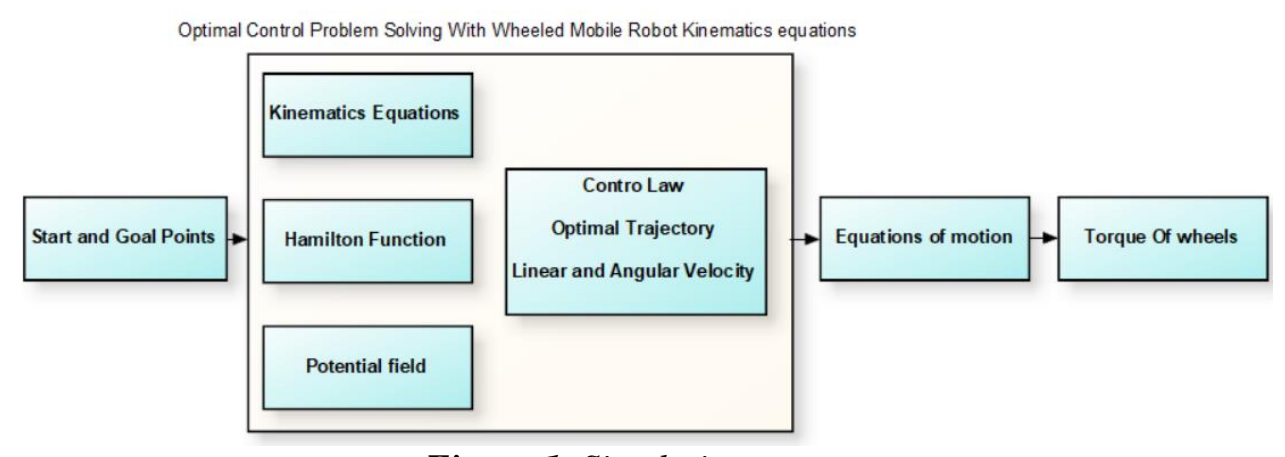

Figure 1. Simulation process

According to Figure 1, by specifying the start and end point, the kinematic equations and the potential function are selected as the input of the Hamilton function, and then by applying mathematical relations, the control law will be obtained.

\section{Kinematics Model for a WMR}

In this paper, a nonholonomic robot model will be considered, which is not equipped with instantaneous motion components in the lateral direction. At moderate speeds of robots, typically, idealized rolling wheel models are employed in which the wheel can only rotate, while no slip is considered in the lateral direction and driving. Consider the WMR, which is shown in Figure 2. Its position and orientation are represented by $\boldsymbol{q}=\left[\boldsymbol{r}^{T}, \lambda\right]^{T}$, in which $\boldsymbol{r}=[x, y]^{T}$ is the position of the WMR's center of mass, and $\lambda$ is the heading angle of the robot. Virtual input $[v, \omega]^{T}$ represents the angular and linear velocities of the robots. The WMR's kinematics model considering the non-slipping, nonholonomic constraints, and pure rolling is presented as follows:

$$
\left[\begin{array}{l}
\dot{x} \\
\dot{y} \\
\dot{\lambda}
\end{array}\right]=\left[\begin{array}{cc}
\cos (\lambda) & 0 \\
\sin (\lambda) & 0 \\
0 & 1
\end{array}\right]\left[\begin{array}{l}
v \\
\omega
\end{array}\right] \triangleq \boldsymbol{J}(\lambda) \boldsymbol{u} .
$$

It can be shown in a general form as the following: 


$$
\dot{\boldsymbol{x}}=\boldsymbol{f}(\boldsymbol{x}, \boldsymbol{u})
$$

where $\boldsymbol{u}$ and $\boldsymbol{J}(\lambda)$ are velocity vector and the full rank velocity transformation matrix, respectively. Also, the radius of each driving wheel and the distance between the centers of the two wheels are shown via $r$ and $b$, respectively. Let us consider that the coordinates of the robot's center of mass are amid the driving wheels, where all the velocity and position variables are time-dependent, and in Equation (1), $\mathbf{u}$ and $\mathbf{x}$ are the control signal and the state of the system, respectively. It is noteworthy that the mobile robot is subject to nonholonomic constraints [12] in which the driving wheels roll exclusively with no slipping, that is:

$$
\dot{y} \cos (\lambda)-\dot{x} \sin (\lambda)=0 .
$$

For the robot to start moving from the initial condition to the target, finding a suitable control signal is a must to prevent collisions with obstacles and minimize the required energy and time.

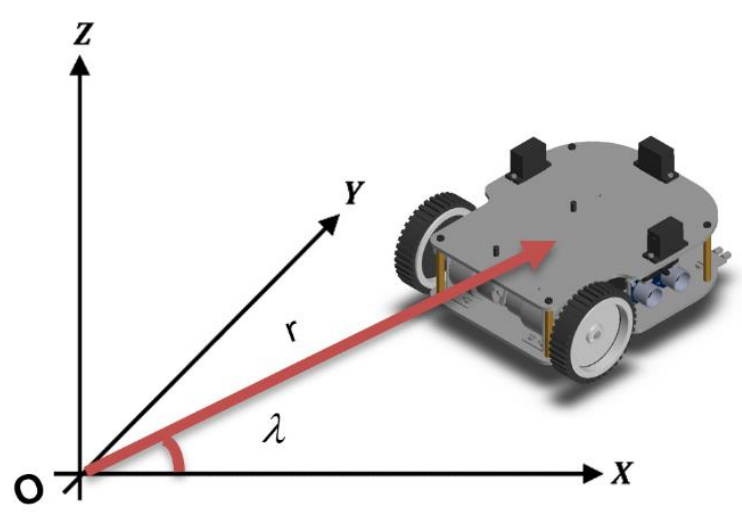

Figure 2. Schematic of wheeled mobile robot

Also, the relationship between torque and the optimal input signal is as follows [12]:

$$
\left[\begin{array}{c}
\dot{v} \\
\dot{w}
\end{array}\right]=\left[\begin{array}{cc}
\frac{1}{m r} & \frac{1}{m r} \\
\frac{L}{2 J r} & \frac{-L}{2 J r}
\end{array}\right]\left[\begin{array}{l}
\tau_{r} \\
\tau_{l}
\end{array}\right] .
$$

\section{THE PROBLEM OF OPTIMAL CONTROL WITH THE OBSTACLE AVOIDANCE}

When planning the nonholonomic mobile robots' optimum motion, the system's kinematic equations are assumed as limitations of the optimal control problem, which involves minimization of time and kinetic energy and prevents collisions with obstacles along the trajectory. In the present investigation, a potential field is used to prevent collisions with obstacles. A mobile robot in the presence of multiple obstacles is shown in Figure 3. One can express a potential field as the sum of the attractive field given the goal point $U_{\text {attr }}(\boldsymbol{r})$ and a repulsive field $U_{\text {rep }}(\boldsymbol{r})$ caused by obstacles [12]:

$$
U(\boldsymbol{r})=U_{\text {attr }}(\boldsymbol{r})+U_{\text {rep }}(\boldsymbol{r})
$$

Attractive potential $U_{\text {attr }}(\boldsymbol{r})$ can be defined as proportional to the squared Euclidean distance to the goal point $\Delta\left(\boldsymbol{r}, \boldsymbol{r}_{\text {goal }}\right)=\left\|\boldsymbol{r}-\boldsymbol{r}_{\text {goal }}\right\|=\sqrt{\left(x-x_{\text {goal }}\right)^{2}+\left(y-y_{\text {goal }}\right)^{2}}$, as follows: 


$$
U_{a t t r}(\boldsymbol{r})=\frac{1}{2} k_{a t t r} \Delta^{2}\left(\boldsymbol{r}, \boldsymbol{r}_{\text {goal }}\right)
$$

where $k_{\text {attr }}$ stands for a positive constant. One can express the repulsive field potential as follows:

$$
U_{\text {rep }}(\boldsymbol{r})=\left\{\begin{array}{cr}
\frac{1}{2} k_{\text {rep }}\left(\frac{1}{\Delta\left(\boldsymbol{r}, \boldsymbol{r}_{\text {obst }}\right)}-\frac{1}{\Delta_{0}}\right)^{2} & \text { if } \Delta(\boldsymbol{r}) \leq \Delta_{0} \\
0 & \text { if } \Delta(\boldsymbol{r})>\Delta_{0}
\end{array}\right.
$$

where $k_{\text {rep }}$ is a positive constant.

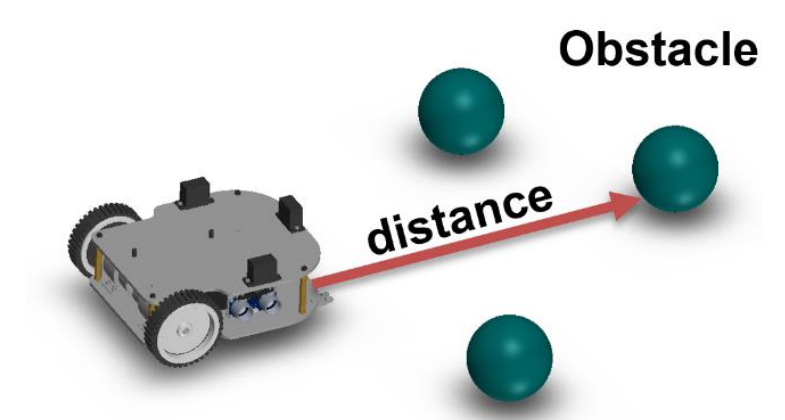

Figure 3. A WMR faced with obstacles

Equation (5) should be considered in the cost function for minimization to prevent collisions with obstacles. The final equation is as follows:

$$
\left\|L_{i}\right\|^{2}=Z_{i} \frac{1}{\left(\operatorname{dist}(t)-r_{m}-r_{o b i}\right)^{2}}
$$

where $Z_{i}$ stands for the weighting coefficient associated with the obstacle avoidance, while $r_{i}$ reflects an enclosed circle radius to the mobile robot. The velocities are squared via basic classical mechanic equations, and the kinetic energy is proportional to some constants. As a result, the parameters to be optimized are $v$ and $\omega$, respectively. In the present study, the principle expressed by Lev Semenovich Pontryagin is used, which is a specific case of the Euler-Lagrange equation [13]. Here, the objective is to detect optimal control vector $\mathrm{u}$ and an optimal state vector $\mathrm{x}$ for the system constraints equations, and also, the objective function is as follows:

$$
\begin{aligned}
& \min _{\boldsymbol{u}(t)}\left\{\phi\left(t_{f}\right)+\int_{0}^{t_{f}} L(\boldsymbol{u}(t)) d t\right\} \\
& \text { suchthat }: \dot{\boldsymbol{x}}(t)=\boldsymbol{f}(\boldsymbol{x}(t), \boldsymbol{u}(t)) \\
& \boldsymbol{x}(0)=\boldsymbol{x}_{0} \\
& \boldsymbol{x}\left(t_{f}\right)=\boldsymbol{x}_{f} \\
& |\boldsymbol{u}(t)|<\boldsymbol{u}_{\max }
\end{aligned}
$$

where $\phi$ is the final state penalty expressed as follows:

$$
\phi\left(t_{f}\right)=\beta t_{f}
$$

where $\beta$ is a weighting parameter and $L(\boldsymbol{u}(t))$ is associated with the kinetic energy of the mobile robot: 


$$
L(\boldsymbol{u}(t))=\boldsymbol{u}(t)^{T} \boldsymbol{u}(t)
$$

It is noteworthy that the appropriate mode of the last state function $\beta$ in Equation (10) is very important; $\beta$ is the weighting coefficient. On the other hand, as $\beta$ is increased, $t_{f}$ becomes more important regarding the integral containing $\mathrm{L}$, which is deemed as the increased significance of minimizing $t_{f}$ more than the remaining terms, and as $\beta$ declines, minimizing the energy rather than the total time becomes more important. By combining the potential field to prevent collisions with obstacles and kinetic energy and time to the function, a new cost function is introduced as the following:

$$
J=\phi\left(t_{f}\right)+\frac{1}{2} \int_{0}^{t_{f}}\left(L(\boldsymbol{u}(t))+\left\|L_{i}\right\|^{2}\right) d t
$$

\section{SOLUTION AND CONDITIONS OF PROBLEM OF THE OPTIMAL CONTROL}

Here, the mathematical details employed for solving the optimal control problem are presented via a numerical technique. By applying the calculus of variations and the Pontryagin minimum principle, the Hamiltonian function is represented as follows:

$$
H(\boldsymbol{x}, \boldsymbol{u}, \boldsymbol{P})=L(\boldsymbol{u})+\boldsymbol{P}^{T} \boldsymbol{f}(\boldsymbol{x}, \boldsymbol{u})=v^{2}+\omega^{2}+P_{1} v \cos (\lambda)+P_{2} v \sin (\lambda)+P_{3} \omega+\left\|L_{i}\right\|
$$

in which $\boldsymbol{P}$ stands for the co-state vector, $\boldsymbol{f}(\boldsymbol{x}, \boldsymbol{u})$ is determined through Equation (1) and Equation (11) gives the $L(\boldsymbol{u})$. The optimality conditions are extracted as a set of differential equations as follows [13]:

$$
\dot{\boldsymbol{x}}^{*}=\frac{\partial H}{\partial \mathbf{P}}=\left[\begin{array}{c}
v^{*} \cos \lambda^{*} \\
v^{*} \sin \lambda^{*} \\
\omega^{*}
\end{array}\right]
$$

and

$$
\begin{aligned}
& \dot{\boldsymbol{P}}^{*}=-\frac{\partial H}{\partial \boldsymbol{x}}\left(\boldsymbol{x}^{*}(t), \boldsymbol{u}^{*}(t), \boldsymbol{P}^{*}(t), t\right) \\
& \boldsymbol{u}^{{ }^{* T}} \boldsymbol{u}^{*}+\boldsymbol{P}^{* T} \boldsymbol{f}\left(\boldsymbol{x}^{*}, \boldsymbol{u}^{*}\right) \leq \boldsymbol{u}^{T} \boldsymbol{u}+\boldsymbol{P}^{*_{T}} \boldsymbol{f}\left(\boldsymbol{x}^{*}, \boldsymbol{u}\right)
\end{aligned}
$$

in which the superscript star refers to as the optimal value of the variables

$$
\frac{\partial H}{\partial \boldsymbol{u}}=\left[\begin{array}{c}
2 v^{*}+p_{1}^{*} \cos \lambda^{*}+p_{2}^{*} \sin \lambda^{*} \\
2 \omega^{*}+p_{3}^{*}
\end{array}\right]=0
$$

From Equation (16) of the optimal control law that includes optimal linear and angular velocities, Equation (16) is obtained as follows:

$$
\begin{aligned}
v^{*} & =-\frac{1}{2}\left(p_{1}^{*} \cos \lambda^{*}+p_{2}^{*} \sin \lambda^{*}\right), \\
\omega^{*} & =-\frac{1}{2} p_{3}^{*} .
\end{aligned}
$$


To solve the free final time $t_{f}$, the problem of optimal control must be transformed into a standard form suggested in [14].

\section{SIMULATION}

The generated path for the case where the starting point is $q_{s}=(5,1,0)$ and the target point is $q_{t}=(10,8,2.5)$ without obstacle is presented in Figure 4.

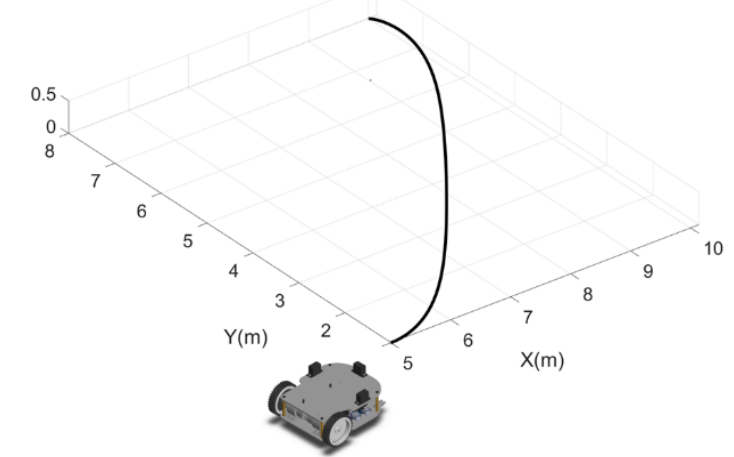

Figure 4. The generated path without Obstalce

The generated path is presented for the case, where the starting point is $q_{s}=(5,1,0)$ and the target point is $q_{t}=(10,8,2.5)$, and there is a constant obstacle with a radius of 0.5 , and the coordinates $x_{o b s 1}=(8,4)$ is presented in Figure 5.

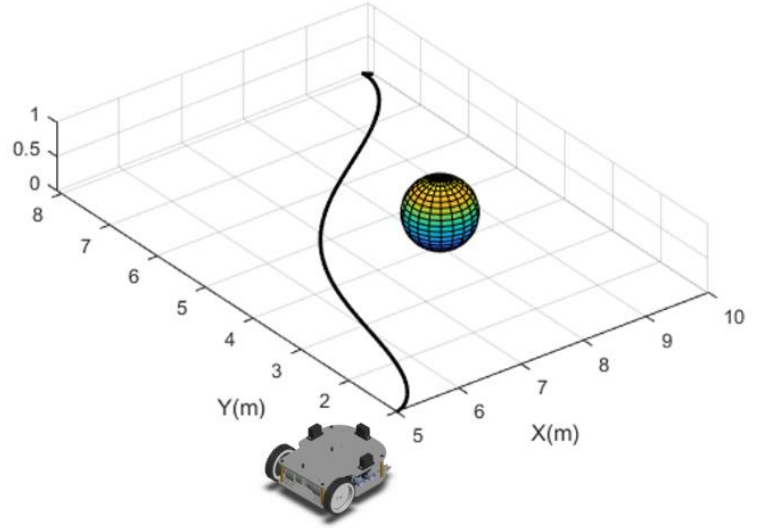

Figure 5. The generated path with one obstacle

The generated path is presented for the case where the starting point is $q_{s}=(5,1,0)$, and the target point is $q_{t}=(10,8,2.5)$, and there are two constant obstacles with a radius of 0.5 , and the coordinates $x_{o b s 1}=(8.5,6)$ and $x_{o b s 2}=(9,3)$ is presented in Figure 6. 


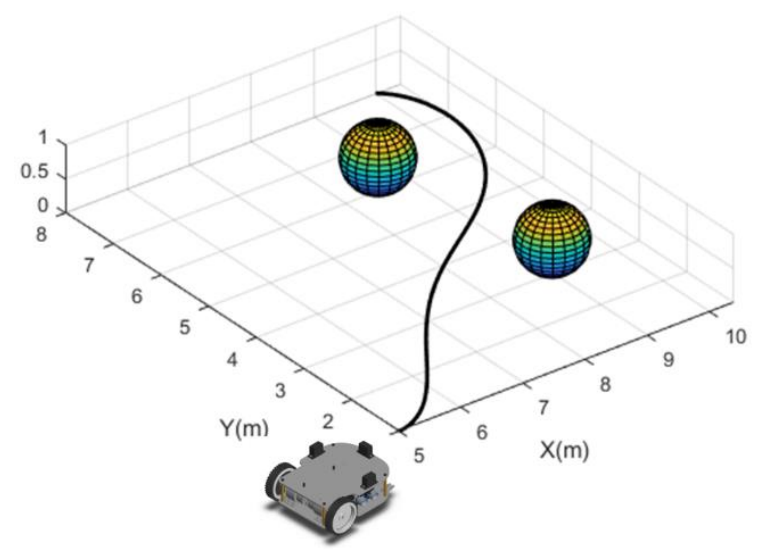

Figure 6. The generated path with multi obstacle

The time is equal to $14.25 \mathrm{~s}, 14.73 \mathrm{~s}, 12.78 \mathrm{~s}$ for states where the path is unobstructed, the path with one obstacle, the path with two obstacles, respectively. Figure 7 shows the position and orientation as a function of time for motions are shown in.

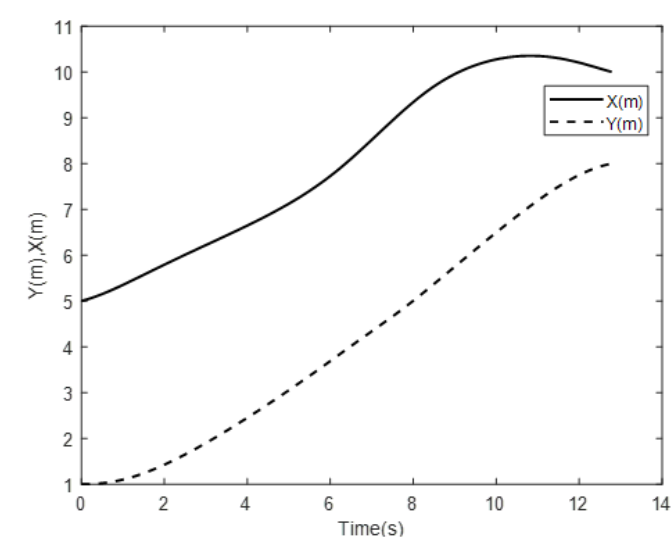

a

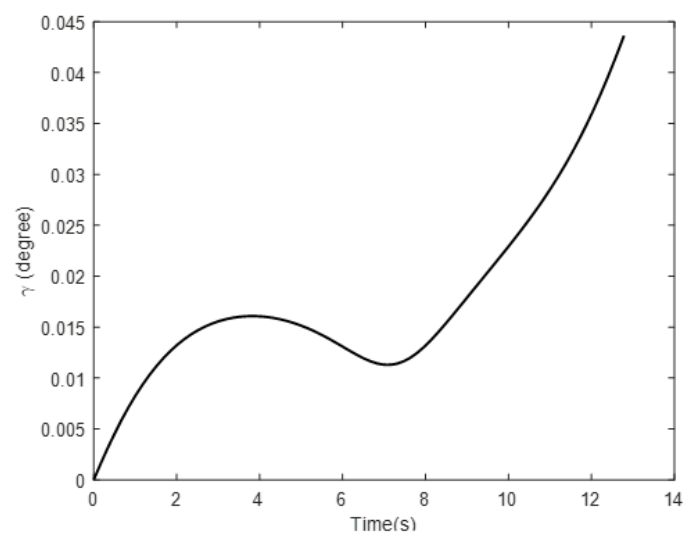

$\mathrm{b}$

Figure 7. (a) Position X, Y of the robot (b) Orientation of the studied robot as a function of time

Figure 8 presents the angular and linear velocities as a function of time for motion

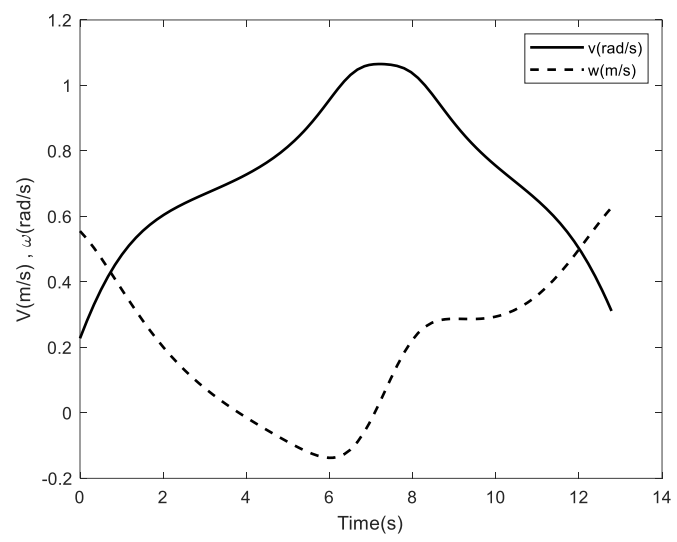

Figure 8. The angular and linear velocity as a function of time

The effect of the $\beta$ on time and cost function is presented in Figure 9 below. 


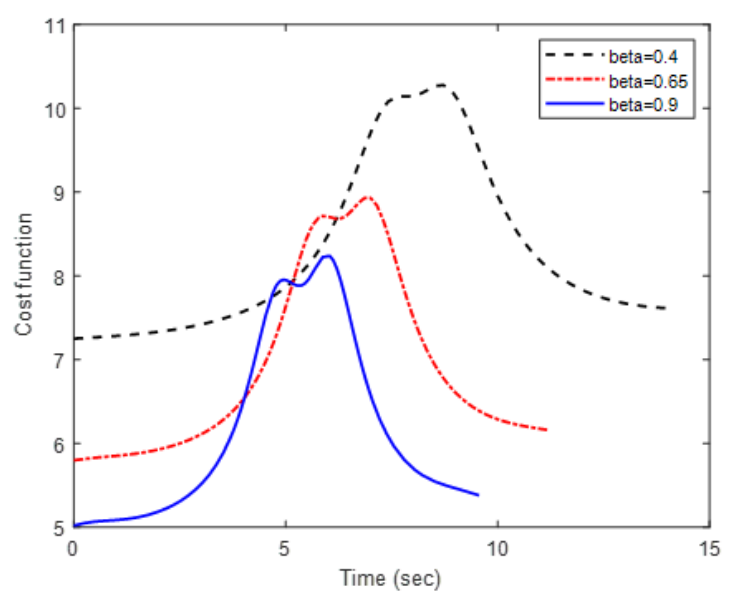

a

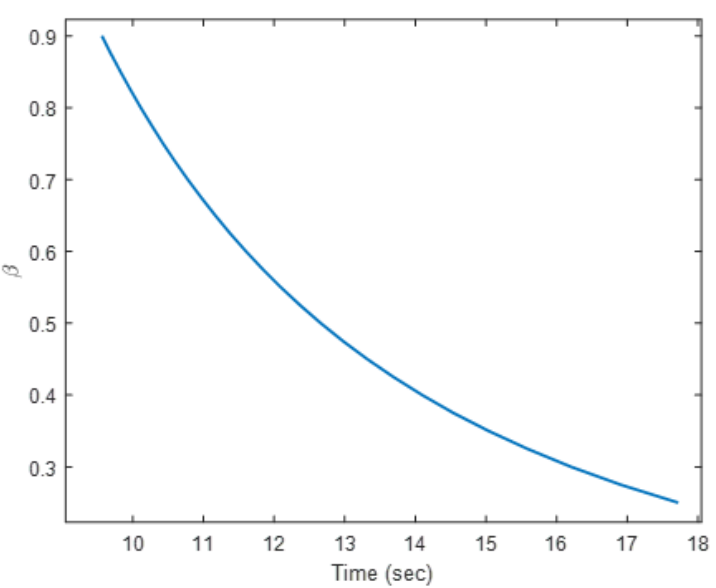

b

Figure 9. (a) The value of the cost function in terms of time for different $\beta(b)$ the effect of $\beta$ on time

The lower and upper limits of the torques for Dc actuators of the mobile robot are obtained as:

$$
\begin{aligned}
& U^{+}=K_{1}-K_{2} v \\
& U^{-}=-K_{1}-K_{2} v .
\end{aligned}
$$

In the simulations, the actuator constants are considered as:

$$
\begin{aligned}
& K_{1}=\left[\begin{array}{ll}
17 & 17
\end{array}\right] \\
& K_{2}=\left[\begin{array}{ll}
1.8 & 1.8
\end{array}\right] .
\end{aligned}
$$

Figure 10 depicts the optimum torque values for the wheel actuators.
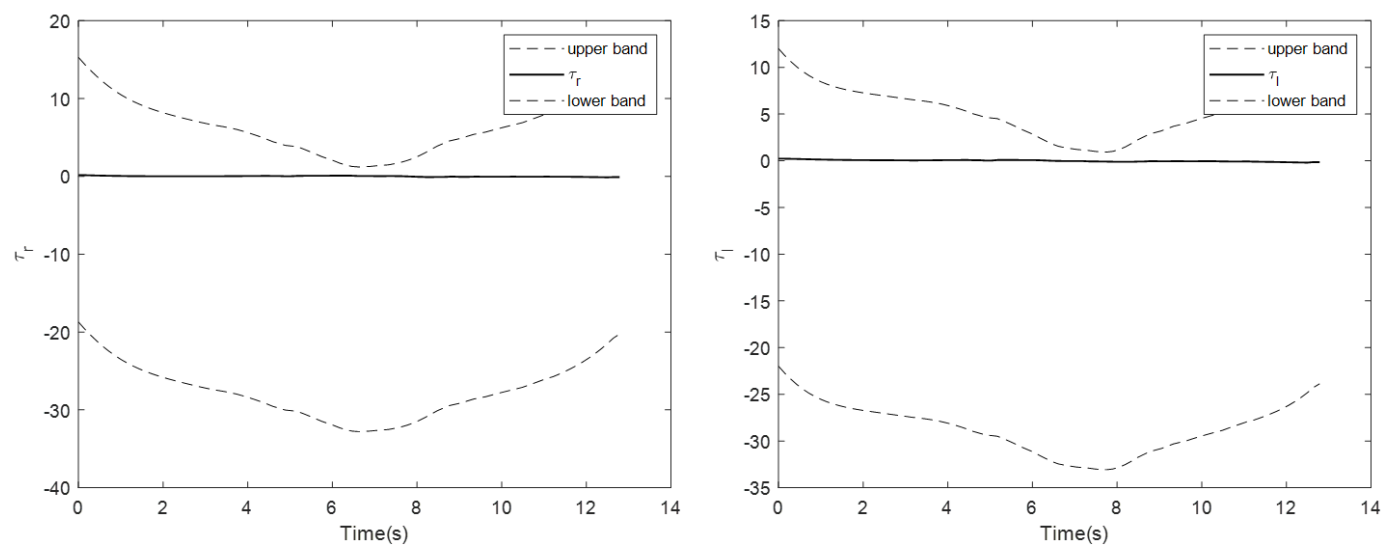

Figure 10. Optimal torques of wheeled (a) right wheeled (b) left wheeled

\section{RESULTS}

In the present research, the path design with obstacle avoidance was investigated for a mobile robot simultaneously by optimizing time and kinetic energy in the presence of fixed obstacles on the basis of open-loop optimal control theory. Also, in many studies, only optimal path design or optimal path design with considered obstacle avoidance have been investigated without paying attention to time and kinetic energy. First, the robot's nonlinear equations were expressed. Then the appropriate cost function was presented for optimum motion control of the system and obstacle avoidance. Cost function includes the time and inputs of velocity and the potential field for obstacle avoidance formulated in optimal control problems. To solve the optimal control problem, the minimum Pontryagin principle has been utilized. In the proposed method, the selection of the $\beta$ parameter is important because it directly affects the time 
optimization, and the effect of the parameter values on time has been determined. At last, a simulation study has been carried out on a mobile robot, and the optimum path without collision in minimum time has been generated, suggesting that the proposed method can be used in practical applications.

\section{CONFLICTS OF INTEREST}

No conflict of interest was declared by the authors.

\section{REFERENCES}

[1] Pandey, A., Pandey, S., Parhi, D.R., "Mobile robot navigation and obstacle avoidance techniques: A review", International Robotics \& Automation Journal, 2(3): 00022, (2017).

[2] Spong, M. W., Hutchinson, S., Vidyasagar, M., "Robot modeling and control”, John Wiley \& Sons, United States, (2020).

[3] Cui, M., Sun, D., Liu, W., Zhao, M., Liao, X., "Adaptive tracking and obstacle avoidance control for mobile robots with unknown sliding", International Journal of Advanced Robotic Systems, 9(5): 171, (2012).

[4] Nazemizadeh, M., Rahimi, H. N., Khoiy, K.A., "Trajectory planning of mobile robots using indirect solution of optimal control method in generalized point-to-point task", Frontiers of Mechanical Engineering, 7(1): 23-28, (2012).

[5] Yen, C.T., Cheng, M.F., "A study of fuzzy control with ant colony algorithm used in mobile robot for shortest path planning and obstacle avoidance", Microsystem Technologies, 24(1): 125-135, (2018).

[6] Liu, J., Yang, J., Liu, H., Tian, X., Gao, M., “An improved ant colony algorithm for robot path planning", Soft Computing, 21(19): 5829-5839, (2017).

[7] Nazemizadeh, M., Mallahi Kolahi, P., "Trajectory Tracking of an Intelligent Mobile Robot on a Slope Surface using the Nonlinear Sliding Mode Control", Mechanic of Advanced and Smart Materials, 1(1): 1-14, (2021).

[8] Korayem, M.H., Ghobadi, N., Fathollahi Dehkordi, S., "Designing an optimal control strategy for a mobile manipulator and its application by considering the effect of uncertainties and wheel slipping", Optimal Control Applications and Methods, 1-25, (2021).

[9] Mallahi Kolahi, P., Mosayebi, M., "Optimal Trajectory Planning for an Industrial Mobile Robot using Optimal Control Theory", Journal of Modern Processes in Manufacturing and Production, 10(3), 25-34, (2021).

[10] Korayem, M.H., Nazemizadeh, M., Nohooji, H.R., "Optimal point-to-point motion planning of nonholonomic mobile robots in the presence of multiple obstacles", Journal of the Brazilian Society of Mechanical Sciences and Engineering, 36(1): 221-232, (2014).

[11] Wu, Y., Liu, L., Yang, Y., Dai, S., "Optimal Control Method for Robot-Tracking Based on Control-Lyapunov-Function”, IEEE Access, 7: 90565-90573, (2019).

[12] Klancar, G., Zdesar, A., Blazic, S., Skrjanc, I., "Wheeled mobile robotics: from fundamentals towards autonomous systems", Butterworth-Heinemann, United Kingdom, (2017).

[13] Kirk, D.E., "Optimal control theory: an introduction", Courier Corporation, Prentice Hall, New York, (1994). 
[14] Wang, X., "Solving optimal control problems with MATLAB: Indirect methods", Industrial and Systems Engineering Department., NCSU, Raleigh, NC, 27695, (2009). 